\title{
General Relativity
}

\section{J. Ehlers}

Max-Planck-Institut für Gravitationsphysik (Albert-Einstein-Institut), Am Mühlenberg 1, 14476 Golm, Germany

Juergen.Ehlers@aei.mpg.de

\section{Introduction}

This introduction is meant to indicate some properties of general relativity theory (GRT) which distinguish it from other branches of physics considered in this book, to relate it to other branches of physics and to mention some of its achievements and open problems. The subsequent chapters will give details.

1.1 GRT is the only empirically supported theory in which the spacetime structure is treated as dynamical, and not specified once and for all, independently of physical processes. Since the spacetime metric is interrelated to matter and field variables via field equations, the distinction between kinematics and dynamics is abolished in GRT.

Conceptually, the background independence must be seen as the principal achievement of GRT; it is, however, at the same time the main obstacle to overcome if GRT and quantum theory are to be united.

1.2 According to GRT the spacetime metric (and the connection and curvature derived from it) represents both the "metric" in the original sense - time, distance, causal order - and the gravitational inertial field; it unifies geometry, chronometry, gravity and inertia. (Einstein: "gravitational field and metric are manifestations of the same physical field".)

1.3 GRT may be viewed as encompassing in a coherent system all of macroscopic, phenomenological physics, from laboratory scales to cosmology.

1.4 So far, all physical theories, classical or quantum, employ a metric to represent matter or fields and their interactions. For this reason GRT is, in principle, a basic ingredient of physics even if gravitation is quantitatively negligible in many contexts. Since inertial mass is inseparable from active, 
gravity-producing mass, an ultimate understanding of mass can be expected only from a theory comprising inertia and gravity.

1.5 Mathematically, GRT is fairly well understood. Several physically interpreted exact solutions to its field equations, with and without matter, are known, as well as general existence and uniqueness theorems [1]. For complex realistic circumstances, perturbation schemes and numerical methods are available. There is, at least in principle, no interpretation problem.

1.6 The existence of a Lorentz metric, the most basic assumption of GRT, implies the approximate validity of special relativity theory (SRT) in spacetime regions which are small compared to the time and distance scale set by the curvature of spacetime. Even in neutron stars this scale is much larger than the scales relevant for the properties of bulk matter, atoms or nuclei. Therefore equations of state, cross sections, transport coefficients etc. derived from quantum theory can be incorporated into the classical matter models used in GRT in spite of the fact that these theories are in principle incompatible.

1.7 So far all experimental tests of GRT have supported the theory [2]. This concerns laboratory experiments which test the existence of a Lorentz metric or, equivalently, of local inertial frames; experiments with clocks, satellites and electromagnetic signals around the Earth and in the solar system, and the dynamics of binary pulsar systems including gravitational radiation damping. GRT has also been used increasingly to analysze and interprete astrophysical and cosmological phenomena. (Here one so far unexplained observation, the "pioneer anomaly" [3], deserves to be mentioned, which has been related tentatively to quintessence [4].)

1.8 At present, gravitational physics is one of the most active areas of research. Great efforts are being made to directly detect gravitational waves, with the prospect to open another window into the universe. Another goal is to find direct evidence supporting the assumption that the large concentrations of mass in the centers of galaxies are indeed black holes. High energy astrophysics offers additional challenges such as the explanation of gamma ray bursts. At a more conservative side, the investigation of gravitomagnetism, opened up by gravity probe B, might be mentioned. This shows that classical GRT is not a closed subject; compared to electrodynamics, gravitational physics has not yet reached the stage of Hertz's experiments.

1.9 The fundamental problem of unifying quantum theory and GRT is considered in other contributions to this book. Here I want to remark only that, in my view, "quantizing general relativity" is a rather inadequate way to address the problem. A unification presumably requires basic changes of quantum theory as well as of GRT, at least if the resulting theory is to remove 
infinites from both theories and to bring light to issues such as non-baryonic dark matter and dark energy.

\section{Basic Assumptions of GRT}

2.1 In GRT as well as in Newtonian physics and SRT, spacetime, the arena of directly perceivable phenomena, is represented as a connected real, 4-dimensional differentiable manifold $M$. This manifold is not generally identified with $\mathbf{R}^{4}$, however. $M$ depends on the situation to be modelled; it can only be determined in connection with a solution to the field equation (see 3.5). $M$ by itself has no physical meaning; it gets meaning only through fields defined on it.

The manifold $M$ is assumed to carry a Lorentz metric $g_{\alpha \beta}$. This assumption guarantees that (i) SRT with its non-gravitational laws remains approximately valid locally even if gravitational fields are taken into account $(2.2,2.3)$; (ii) the connection $\Gamma_{\beta \gamma}^{\alpha}$ (or covariant derivative operator $\nabla_{\alpha}$ ) determined by $g_{\alpha \beta}$ provides a natural way to express the influence of gravity on "matter" $(2.4-2.6)$; and (iii) the interaction between matter and gravity can be expressed via the curvature associated with $\Gamma_{\beta \gamma}^{\alpha}(2.7)$. Here "matter" is used to denote all physical entities besides $g_{\alpha \beta}$, i.e. everything which carries localizable energy and momentum.

The beauty of GRT is due to the fact that one mathematical object, the metric field $g_{\alpha \beta}$ and fields derived from it, provides all three aspects of gravity listed above, without the need to introduce additional structures.

On the other hand, the division of physical entities into the metric and "everything else" calls perhaps for a more democratic or, even better, a monistic structure which, however, apparently is not in sight.

2.2 Given a point ("event") $x$ on a spacetime $\left(M, g_{\alpha \beta}\right)$, there exists a coordinate system $\left(x^{\alpha}\right)$ on a neighbourhood $N$ of $x$ such that $x^{\alpha}=0$ at $x$ and, on $N$,

$$
g_{\alpha \beta}\left(x^{\varepsilon}\right)=\eta_{\alpha \beta}+p_{\alpha \gamma \beta \delta}\left(x^{\varepsilon}\right) x^{\gamma} x^{\delta}
$$

where $\eta_{\alpha \beta}=\operatorname{diag}(1,1,1,-1)$, the functions $p$ have the symmetries of the curvature tensor $R_{\alpha \gamma \beta \delta}$ associated with $g_{\alpha \beta}$, and $p_{\alpha \gamma \beta \delta}(0)=-\frac{1}{3} R_{\alpha \gamma \beta \delta}(0)$. For fixed $x$, such "normal" coordinates are unique up to Lorentz transformations 1

\footnotetext{
${ }^{1}$ The statements about normal coordinates are equivalent to a coordinateindependent fact: a neighbourhood of the zero vector of the tangent space at $x$ can be mapped diffeomorphically onto a neighbourhood of $x$ in $M$ such that straight lines go into geodesics starting at $x$. In spite of their usefulness in GRT, (1) and (2) are rarely mentioned; I found it only in Pauli's relativity article in the Encyclopedia of Mathematics and Mathematical Sciences. The theorem is true for arbitrary dimensions and signatures; it holds if the metric is $C^{2}$. A proof has recently been given by B.G. Schmidt (unpublished) .
} 
These coordinates satisfy

$$
(\text { at } x): g_{\alpha \beta}=\eta_{\alpha \beta}, g_{\alpha \beta, \gamma}=0, g_{\alpha \beta, \gamma \delta}=\frac{2}{3} R_{\alpha(\gamma \delta) \beta}
$$

Coordinates obeying the first line of (2) are called "locally inertial at $x$ "; normal coordinates form a subclass of them 2 The existence of normal coordinates indicates that any Lorentz metric can be approximated by the flat Minkowski metric $\eta_{\alpha \beta}$ in a region small compared to the curvature scale, $\max \left|R_{\alpha \gamma \beta \delta}\right|^{-1 / 2}$, and (2) identifies the curvature tensor as a measure of an "intrinsic" gravitational field, i.e. one that cannot be "transformed away" by a coordinate change.

2.3 As a global restriction on physical spacetimes one assumes the manifold $M$ to be orientable and $\left(M, g_{\alpha \beta}\right)$ to be time-oriented. This last property means that there exists a continuous, never-vanishing timelike vector field which is said to point into the future. Timelike and lightlike vectors pointing into the same half of the null cone as that specified vector are then also called future pointing. These (rather weak) global restrictions are made to give meaning to the discrete symmetry operations $T$ (time reversal) and $P$ (parity), and to formulate local laws which presuppose a time-orientation such as the second law of thermodynamics, molecular chaos, or the quantum law for transition probabilities.

2.4 The existence of normal coordinates suggests the transfer of local physical laws from special to general relativity: formulate the law in SRT as a tensor equation with respect to inertial coordinates and substitute $g_{\alpha \beta}, \nabla_{\alpha}$ for $\eta_{\alpha \beta}$, $\partial_{\alpha}$, respectively, to obtain a tensorial GRT law. This law is seen to be identical to the original law at the origin $x$ of any normal coordinate system, hence it will differ from its ancestor very little in a sufficiently small neighbourhood of any event $x$.

This rule is unambiguous if the SRT-law is algebraic or of first differential order. It provides a physical interpretation of the metric and the matter variables involved. The consistency of the laws so obtained is not implied by the rule itself 3

Simple consequences of this hypothesis are Einstein's generalized law of inertia freely; falling test particles have timelike geodesic world lines given by

\footnotetext{
${ }^{2}$ Given a timelike geodesic $G$, it is also possible to introduce local coordinates in a neighbourhood of $G$ such that $G$ is the "spatial origin", and such that the first two equations of (2) are valid on $G$. Such coordinates are "locally inertial on G" and represent Einstein's elevator better that those defined in the text. It is instructive to consider how "freely falling test masses" contained in a dragfree satellite realize, as precisely as possible, geodesics enclosed in a local inertial frame.

${ }^{3}$ Examples where difficulties arise have been discovered by H.A. Buchdal, G. Velo and D. Zwanziger. For discussion and refs. see, e.g., [5].
} 


$$
\ddot{x}^{\alpha}+\Gamma_{\beta \gamma}^{\alpha} \dot{x}^{\beta} \dot{x}^{\gamma}=0 ;
$$

ideal clocks measure proper time $\int\left|g_{\alpha \beta} d x^{\alpha} d x^{\beta}\right|^{1 / 2}$ along their (not necessarily geodesic) world line; light rays in vacuo correspond to lightlike geodesics.

The rule also supplies GRT-laws for classical matter models including kinetic theory and hydro-, elasto-, thermo- and electrodynamics. These matter models each contain an energy-momentum tensor $T^{\alpha \beta}$. The total energymomentum tensor obeys, in agreement with the correspondence rule $S R T \rightarrow$ $G R T$, the law

$$
T_{; \beta}^{\alpha \beta}=0
$$

which, because of the covariant derivative, is not a conservation law in the ordinary sense. This comes as no surprise since the gravitational field acts on matter. (See 3.8.)

The considerations of this section, which concern non-gravitational matter laws in gravitational fields, may be taken as an exact expression of (many formulations of) Einstein's heuristic "principle of equivalence".

2.5 Energy is usually asumed to be positive and to dominate stresses. Accordingly, $T^{\alpha \beta}$ is said to be energy dominated if its components with respect to any orthonormal basis satisfy

$$
T^{00} \geq\left|T^{\alpha \beta}\right|
$$

for all $\alpha, \beta$.

Hawking [6] has shown: if (4) and (5) hold, and if $T^{\alpha \beta}=0$ on a compact part $S$ of a spacelike hypersurface, then $T^{\alpha \beta}=0$ in the domain of dependence of $S$. Thus, matter obeying (4) and (5) cannot move faster than light into an empty region, since otherwise it could enter the domain of dependence of $S$ from the outside of $S$. This result is remarkable, since (4) represents 4 equations for 10 unknowns; without (5) the conclusion does not hold.

2.6 In GRT the concept "free particle" is abandoned since all matter seems to be universally coupled to gravity. Accordingly the law of inertia is replaced in GRT by the geodesic law (3) to represent free fall. No concept of mass enters that law (or its predecessor, Galileo's law), though for historical reasons it is said to express the universal proportionality (or equality) of inertial and gravitational mass.

It follows from (3) that the relative position vector $r^{\alpha}$ of two infinitesimally close (in the sense of a variation), freely falling particles obeys the equation of geodesic deviation

$$
\ddot{r}^{\alpha}=R_{\beta \gamma \delta}^{\alpha} \dot{x}^{\beta} \dot{x}^{\gamma} r^{\delta}
$$

where the dot indicates covariant differentiation with respect to the proper time of one of the geodesics $\dot{x}^{\alpha}(\tau)$. 
This equation characterizes the curvature tensor. It shows that the ordinary law of inertia, if expressed in terms of relative motions, holds, within the framework of Lorentzian spacetimes, if and only if the spacetime is flat, and it provides the interpretation of the curvature tensor as the gravitational tidal field.

2.7 So far, the assumptions which have been introduced hold in any "metric" theory of spacetime including SRT, since no field equation has been imposed on $g_{\alpha \beta}$.

To obtain a field equation relating $g_{\alpha \beta}$ to matter, Einstein assumed, in analogy to Poisson's law, an equation of the form

$$
V^{\alpha \beta}\left(g_{. .}, \partial g_{. .}, \partial^{2} g_{. .}\right)=\kappa T^{\alpha \beta}
$$

where the l.h.s. is a tensor-valued function depending on the arguments indicated; it is assumed to be linear in the second derivatives $g_{\alpha \beta, \gamma \delta}$.

Remarkably these assumptions determine $V^{\alpha \beta}$, as follows. Equation (2) shows: A function like $V^{\alpha \beta}$ can be expressed algebraically in terms of $g_{\alpha \beta}$ and $R_{\alpha \beta \gamma \delta}$ (specialize to the origin of normal coordinates), linearly in $R_{\alpha \beta \gamma \delta}$. Therefore $V^{\alpha \beta}$ must be a linear combination of $R^{\alpha \beta}, g^{\alpha \beta}$ and $R g^{\alpha \beta}$ with constant coefficients. Hence, the looked-for field equation is equivalent to the "tracefree equation"

$$
R_{\alpha \beta}-1 / 4 g_{\alpha \beta} R=\kappa\left(T_{\alpha \beta}-1 / 4 g_{\alpha \beta} T\right)
$$

and a relation involving the traces $R, T$.

Equation (7a), the contracted Bianchi identity, and (4) imply that $R+\kappa T$ is constant. Putting

$$
R+\kappa T=4 \Lambda
$$

gives Einstein's gravitational field equation

$$
R^{\alpha \beta}-1 / 2 R g^{\alpha \beta}+\Lambda g^{\alpha \beta}=\kappa T^{\alpha \beta}
$$

Equation (7a) may be considered as that part of the gravitational field equation which is independent of the "mechanical" or "matter" law (4), while (7b) expresses the compatibility of (7a) with (4). In this argument $\Lambda$ appears as an integration constant.

In 1915, Einstein had assumed in addition that the Minkowski metric should satisfy the vacuum field equation. Then $\Lambda=0$. In 1917 he added $\Lambda$ to allow for a static model of the universe with pressureless matter. For a discussion of the present views on $\Lambda$ in physics and cosmology, see Part VII.

By construction, the field equation (7) implies the energy-momentum law (4). Thus (7) accounts both for the inertia of matter and for its power to attract gravitationally. The constant $\kappa=8 \pi G c^{-4}$ is chosen such that for 
weak fields and slowly moving and weakly stressed matter, Newton's theory emerges as an approximation [7] ( $c=$ speed of light, $G=$ Newtons's constant of gravity).

Just as in Newtonian gravity the Poisson equation contains the trace of the tidal field tensor, so in GRT (7) contains a "trace" $R^{\alpha \beta}$ of the curvature tensor.

\section{General Comments on the Structure of GRT}

3.1 The field equation (7) has physical meaning only if $T^{\alpha \beta}$ is specified; this specification always contains the metric. Mathematical studies often consider the vacuum case, $T^{\alpha \beta}=0$, with or without $\Lambda$. Matter models studied in some detail include perfect fluids, electromagnetic fields, collisionless particle systems idealized by kinetic theory and, to a lesser extent, elastic bodies. In these cases the system of partial differential equations consisting of (7) and the relevant matter law admits a (locally) well-posed initial value problem.

A model of a physical system in GRT thus consists of a structure $\left(M, g_{\alpha \beta}, m\right)$, where $m$ stands for matter variables. Two such models are physically equivalent if their underlying manifolds can be smoothly and bijectively mapped onto each other such that the fields $g_{\alpha \beta}, m$ of one model are mapped into those of the other one. Ideally, a particular model should be characterized by invariant properties. For example, Einsteins's static universe is characterized as the only static solution of (7) with pressureless matter, with the density $\rho$ being the only independent invariant.

3.2 Contrary to appearance, the Einstein equation (7) does not imply matter to be the source that determines the gravitational potential $g_{\alpha \beta}$, for only (at least) the pair $\left(T^{\alpha \beta}, g_{\alpha \beta}\right)$ describes matter, not $T^{\alpha \beta}$ by itself. Equation (7) states a mutual inter-action between metric and matter.

3.3 Equation (7) is incompatible with point particles as matter models. For static, stellar models the mass/radius ratio has an upper bound $c^{2} / 2 G$. The simplest "objects" of GRT which may be taken to replace mass points are black holes, see (4.5) below.

3.4 The tensors in (7) are symmetric. This follows from Einstein's assuptions stated in 2.6. The symmetry of the total energy-momentum tensor is, therefore, essentially a consequence of the assumption that gravity is completely represented by $g_{\alpha \beta}$ and fields derived from it. The same holds for the special kind of non-linearity ("self interaction") of the l.h.s. of (7).

3.5 A solution of (7) is usually constructed in some local coordinate system. Frequently the components $g_{\alpha \beta}$ in that system exhibit singularities. These 
may either be due to the choice of coordinates or to the existence of an intrinsic singularity. A solution can be considered as fully understood only if it has been maximally extended. A maximal solution may be free of singularities; otherwise its (suitably defined) boundary will be singular. The problems of finding maximal extensions and/or characterizing singularities are difficult; we know examples, but no general theorems.

3.6 The background independence, mentioned already in 1.1 , is an important characteristic of GRT. Its meaning is not properly grasped by "general covariance", i.e. the possibility to formulate the laws such that arbitrary local coordinates may be used; that can be done for SRT as well as for Newton's theory. Rather, "absence of background" means that the laws of GRT, in contrast to those of Newtonian physics and SRT, do not presuppose the existence of an "absolute" spacetime structure which is specified categorically prior to dynamical laws and not influenced by physical processes.

In GRT the metric is said to be "dynamical". This involves two interrelated aspects: (i) a $g_{\alpha \beta}$-field is specifiable by independent initial data ("has degrees of freedom") which determine, together with matter data, its evolution (see Sect. 4.3), (ii) the $g_{\alpha \beta}$-field not only acts on matter as, e.g., via (4), but interacts with matter, (7).

The history of physics shows that some essential changes in the foundation of theories consisted in substituting dynamical structures for absolute ones. It appears to be generally accepted that a fundamental theory should be free of any background structure, i.e. its basic structures, not only those of spacetime, should be dynamical, not absolute ones. One might call this the "Mach-Einstein principle".

Historically, the formulation of a theory directly identified its absolute structures. Systematically and in general, it is difficult (if at all possible) to identify these structural elements of a theory unambiguously, especially since a theory may be based on different basic concepts. If, however, the variables and laws to be taken as basic are specified, the distinction absolute/dynamical is unambiguous, in my view.

The issue briefly considered here, and its relation to the principles of general covariance, general relativity and diffeomorphism invariance, is discussed carefully in the next chapter by D. Guilini. For a related discussion from a different viewpoint, see [8], Sects. 2.2.5 and 2.3.

3.7 The assumptions introduced in Sect. 2 are not independent. That light rays are given by lightlike geodesics, e.g., can be deduced from the generally covariant Maxwell equations, the geodesic law can be deduced from (4), (5), and a definition of "test particle", and dynamical clock models can be shown to exhibit proper time. It appears that GRT is semantically consistent, though a complete axiomatic has not been given. 
3.8 As remarked in 2.3, (4) is not a conservation low; integration cannot transform it into a statement saying that the amount of energy contained in some finite volume changes only in accordance with a flux through the boundary. This fact cannot be remedied by adding to the matter energy tensor a gravitational energy tensor; according to GRT, such a tensor does not exist. The reason is simple: The state of a gravitational field, i.e. its Cauchy data, is given by some components of $g_{\alpha \beta}$ and its first partial derivatives. From these data at a point one cannot construct a tensor as required.

It $i s$ possible to find non-tensorial energy-momentum "complexes" which, added to $T^{\alpha \beta}$ (or to its densitized version), obey ordinary divergence equations in consequence of (7), and which give rise to non-tensorial integral conservation laws. Such complexes and laws are used in connection with approximation methods to express GRT-relations in familiar energy terms. It is possible, however, to describe all observable relations of GRT without such non-covariant tools.

In contrast to energy-momentum, scalar quantities like electric or baryonic charges do admit "decent" local and integral conservation laws since scalars at different events can be added unambiguously while vectors cannot.

\section{Theoretical Developments, Achievements and Problems in GRT}

4.1 Einstein's gravitational field equation (7) is the Euler-Lagrange equation associated with the action functional (with $c \equiv 1$ )

$$
A_{D}[g, m]=\int_{D}\left\{\frac{1}{2 \kappa}(R(g)-2 \Lambda)+L(g, m)\right\} d V
$$

in which $D$ denotes a compact domain of spacetime, $g$ stands for the metric, $m$ for matter variables and $d V=\sqrt{\left|\operatorname{detg}_{\alpha \beta}\right|} d^{4} x$ is the invariant volume element of spacetime. Up to a divergence, the curvature scalar is the only invariant function of the metric and its derivatives (of any order) whose variational derivative is of second order in the metric. Up to a divergence, $R$ is a quadratic form in the connection coefficients. The action density $L$ of matter contains the metric and the connection, but not the curvature. The energy tensor is obtained as the variational derivative of $L$,

$$
\frac{1}{2} T^{\alpha \beta}=\frac{1}{\sqrt{|g|}} \frac{\partial(\sqrt{|g|} L)}{\partial g_{\alpha \beta}}
$$

Varying $A$ with respect to $g$ gives (7); varying it with respect to $m$ gives the matter equations. These statements summarize the mathematical contents of Chap. 2 if the appropriate expressions for $L$ are chosen.

For first-order Lagrangian field theories in SRT, the rule for generalizing them to GRT stated in 2.3 is equivalent to the simple device of 
substituting $g$ and $\nabla$ for $\eta$ and $\partial$ in the matter action density. This prescription includes that no curvature term should be introduced into the matter action; this minimal coupling rule may be considered as a version of Einstein's equivalence principle. In this form the principle can be applied not only to the classical matter models mentioned in 2.3 , but also to the formally classical, Lagrange-based standard model of particle physics. That requires, however, that spacetime is considered as the base manifold of a principal fibre bundle with structure group $U(1) \times S U(2) \times S U(3)$; see Part II.

The action (8) is also the starting point for Hamiltonian formulations of gravity, either in terms of metric variables or connection variables (see 4.2). These formulations make it possible to introduce canonical variables and to try canonical quantization of gravity.

4.2 In the standard model of particle physics, principal connections play the part of mediating interactions between massive particles. But although GRT was the first theory in which a connection appeared, besides objects related to linear representations of an underlying group, and the name "gauge" derives from Weyl's attempt to unify electromagnetism and gravitation, GRT is not a pure gauge theory since the gravitational connection $\Gamma_{\beta \gamma}^{\alpha}$ is not a basic field, but is derived from the metric. This is related to the fact that, in contrast to pure gauge theories, the points of the fibres of the $\mathrm{SO}(3,1)$ bundle over $M$ are orthonormal frames of $(M, g)$; the bundle space is said to be soldered to the base space. This special role of the spacetime connection shows up in the gravitational Lagrangian density $R=g^{\alpha \beta} R_{\alpha \beta}$, which is linear, not quadratic, in the curvature like that of Maxwell and Yang-Mills fields. It appears that this is another characteristic feature of gravity which distinguishes it from the other fundamental interactions. In gravity, the gauge potential $\Gamma_{\beta \gamma}^{\alpha}$ itself derives from a potential, the metric.

A historical remark: the procedure

$$
g_{\alpha \beta} \rightarrow \Gamma_{\beta \gamma}^{\alpha} \rightarrow R_{\beta \gamma \delta}^{\alpha}
$$

consisting of two non-linear steps of first differential order leads from a tensor via a connection to a tensor. The impossibility to form tensors from $g_{\alpha \beta}$ by differentiation without the intervention of a non-tensorial field was one of the obstacles Einstein had to overcome on his arduous way to his general theory of relativity. Connections as quantities independent of a metric were introduced only in 1918 by J.A. Schouten and H. Weyl after T. Levi-Civita's introduction of metric connections in 1917. While only the second step in (10) occurs in gauge theories, the first step is peculiar to gravity; it was a step which enabled Einstein to make the metric dynamical and to identify it with (a new kind of) gravitational potential.

4.3 An essential test for the viability of a classical field theory is whether its field equations admit a well-posed initial value problem. Solving the Cauchy 
problem requires identifying initial data and, thereby, states and degrees of freedom, as well as to determine the dependence of the evolved field on its data, i.e. the causal behaviour of the field.

Carrying out this analysis for Einsteins's field equation (7), without or with coupling to matter, turned out to be difficult for reasons which can all be traced back to diffeomorphism invariance. Here I report only the main results without technical details.

The equations split into two subsets. One of these imposes conditions, usually in the form of non-linear elliptic partial differential equations, on the initial data specified on a 3-dimensional Riemannian space (constraint equations). The free data for the gravitational field turn out to correspond to two degrees of freedom per space point, as in the case of ordinary electromagnetism. The second subset consists of the evolution equations. After imposition of coordinate conditions, these turn out to be hyperbolic, non-linear wave equations. For all matter models mentioned in this survey 4 classical ones as well as Dirac and Yang-Mills fields, the outermost characteristics turn out to be lightlike hypersurfaxes, i.e. wave fronts propagating with fundamental speed $c$. This expresses Einstein causality.

Hyperbolicity means the following: the laws imply relations between the fields within finite domains of spacetime, relations which are not affected by the fields outside that domain. The laws, and data on a compact part $\mathrm{S}$ of space, uniquely determine the fields in the (finite) domain of dependence of $S$. This kind of determinism is fundamentally different from that of Laplace which requires data on the whole, infinite space at one instant.

A further important fact is that the first set is preserved under the evolution. Thus, later states of the field again satisfy the so-called "constraint equations". Finally, irrespective of coordinate conditions, the evolved field is determined by the data uniquely up to diffeomorphisms in the domain of dependence of the data.

Spacetimes determined by initial data are said to be globally hyperbolic; their manifolds $M$ are products of a 3-manifold "space" and a 1-manifold "time". The initial value problem for the vacuum field equation with $\Lambda=0$ has been used to prove the existence of global, singularity free, asymptotically flat spacetimes filled with gravitational radiation only. Such solutions arise from initial data close to trivial data giving flat spacetime. They describe how incoming gravitational radiation scatters on itself and propagates out again. Theorems about global solutions with $\Lambda$ are also known.

4.4 An important task for any gravitation theory it the modelling of an isolated system such as a single star, the solar system or a binary star system far removed from other bodies. All quantitative tests of the field equation (7)

\footnotetext{
${ }^{4}$ For models of bulk matter such as fluids, equations of state have to be restricted, however, to exclude, e.g., superluminal sound waves. This holds in SRT already.
} 
are based on approximate solutions to such spacetimes. In this subsection we put $\Lambda=0$.

One expects the spacetime of an isolated system to resemble flat spacetime at large distance from the bodies. To express that asymptotic behaviour R. Penrose proposed to rescale the metric $g_{\alpha \beta} \rightarrow \Omega^{2} g_{\alpha \beta}$ and to let $\Omega$ tend to zero at large physical distances such that one can attach a "boundary at infinity" where $\Omega=0, \Omega_{, \alpha} \neq 0$. The boundary consists of ideal endpoints of outgoing and incoming light rays, respectively, and of spacelike infinity. Such spacetimes may contain outgoing and/or incoming gravitational and electromagnetic radiation. Some exact implications of the vacuum field equation about the asymptotic behaviour of such radiation have been derived, but the motion of bodies emitting radiation so far is the domain of analytical, post-Newtonian approximations and, increasingly, numerical relativity.

For asymptotically flat spacetimes a constant total 4-momentum at spacelike infinity has been defined, and a celebrated result says that it is futuredirected timelike; so it has positive energy (except, of course, for Minkowski spacetime, where it is zero), provided $T^{\alpha \beta}$ is energy dominated. A total 4momentum at null infinity whose (positive) energy decreases towards the future according to the amount of the outgoing radiation has also been defined.

4.5 The vacuum field equation with $\Lambda=0$ has asymptotically flat, particle like solutions, black holes. Their stationary states are characterized by only three parameters, namely mass, angular momentum and charge. The outer part of a black hole spacetime, connected to infinity, is separated from an interior part by a horizon which acts as a one-way membrane: test particles and radiation can pass through from the outside only, not from the inside.

A thermodynamic of black holes has been elaborated [9]; its relation to statistical mechanics, quantum and string theories is a subject of current research.

In astrophysics, black holes are considered as objects which may form when a massive star collapses at the end of its thermonuclear evolution. They are also thought to exist at the centres of most galaxies. Efforts are being made to observe spectroscopic features characteristic of the geometry near a horizon.

4.6 Light cones not only govern the propagation of electromagnetic and gravitational radiation, but they determine causal relations, too. While in flat spacetime light cones are, apart from their vertices, smooth hypersurfaces, in curved spacetimes they have self-intersections and caustics. Observationally these geometric properties show up as the phenomena of gravitational lensing [10]. Distant galaxies, e.g., are observed in different images which differ in brightness and shape. Modelling such phenomena has become a useful tool in astronomy for determining the masses and mass distributions of the deflecting matter including dark matter. The successes of such modelling provide direct evidence for spacetime curvature. They support the light deflection measure- 
ments which followed, with ever increasing accuracy, the famous solar eclipse measurements of 1919 .

4.7 Relativistic celestial mechanics which began with Einstein's perihelion paper of 1915 has been much developed since about 1980, in the form of postNewtonian dynamics whose approximate equations of motion now include corrections of Newton's laws of order up to $\left(\frac{v}{c}\right)^{7}$, where $v$ is a typical relative speed, e.g., in a binary system.

This theory, or rather its first post-Newtonian version which includes only $\left(\frac{v}{c}\right)^{2}$ corrections, has been used to test whether GRT-predictions of relations between observable parameters agree with real observations made on binary systems composed of neutron stars. So far, agreement prevails, which is very remarkable in view of the precision of the data. The predictions include the slowing down rates of the orbital periods, and the agreement with measured values has given indirect evidence for the existence of gravitational waves.

The higher-order approximations are applied tentatively to late stages of compact binaries when the components approach each other ever closer until they "plunge" together to form a single object, perhaps a black hole. Such processes are thought to emit bursts of gravitational radiation which might be detected by gravitational wave interferometers which have started to operate.

\section{Selected References}

1. Schmidt, B.G. (ed.), Einstein's Field Equations and Their Physical Applications, Lect. Notes Phys. 540, Springer 2000. 92

2. Will, C.M., Theory and Experiment in Gravitational Physics, CUP, 1993. 92

3. Anderson, J.D. et al., Study of the anomalous acceleration of Pioneer 10 and 11, Phys. Rev. D 65082004 (2002) and gr-qc/0104064, March 2005. 92

4. Mbelek, J.P., General Relativity and Quintessence Explain the Pioneer Anomaly, gr-qc/0402088, 2006. 92

5. Frauendiener, J., On the Velo-Zwanziger Phenomena, J. Phys. A 36, 8433, 2002. 94

6. Hawking, S.W. and Ellis, G.F.R., The Large Scale Structure of Space-Time, CUP, 1973. 95

7. Ehlers, J., Newtonian Limit of General Relativity, pp. 503-509 in Encyclop. of Mathem. Physics 3, Francoise, J. J. et al. (eds.), Elsevier, Amsterdam, 2006. 97

8. Rovelli, C. Quantum Gravity, (Cambridge University Press, Cambridge, 2004). 98

9. Wald, R.M., Quantum Field Theory in Curved Spacetime and Black Hole Thermodynamics, University of Chicago Press, 1994. 102

10. Schneider, P., Ehlers, J. and Falco, E.E., Gravitational Lenses, Springer, 1992. 102

In addition, the following references - not cited in the text - provide perspective and detailed references: 
Hawking, S.W. and Israel, W. (eds.) 300 Years of Gravitation, CUP, 1987. Classical and Quantum Gravity, 16, No. 12A, 1999.

Straumann, N., General Relativity, Springer, 2004.

Monas, L. and Diaz-Alonso J. (eds.), A Century of Relativity Physics, AIP Conference Proc., N.Y. 2006. 